\title{
Modeling the motion of a Taylor bubble in a microchannel through a shear-thinning fluid
}

\author{
Andrea Aquino ${ }^{1, *}$, Davide Picchi $^{1}$, and Pietro Poesio ${ }^{1}$ \\ ${ }^{1}$ Department of Mechanical and Industrial Engineering, University of Brescia, Brescia 25123, Italy
}

\begin{abstract}
Applications of multiphase flows in microchannels as chemical and biological reactors and cooling systems for microelectronic devices typically present liquid slugs alternated with bubbles of elongated shape, the Taylor bubbles. These occupy almost entirely the cross-section of the channel and present a hemispherical front and a liquid layer, the lubrication film, which separates the gas from the tube wall. The Taylor bubble perturbs the surrounding fluids activating many transport mechanisms in the proximity of the gas-liquid interface; therefore, the bubble motion significantly influences the heat and mass transfer rates. Although many works deeply investigate the bubble hydrodynamics in Newtonian fluids, the knowledge about the relation between bubble hydrodynamics and rheological properties is insufficient, and studies where the continuous phase exhibits a shear-thinning behavior are missing. Our numerical analysis tries to fill this gap by investigating the motion of a Taylor bubble in a non-Newtonian shear-thinning fluid, modeled by the Carreau viscosity model. First, we validate the results against the Newtonian case and a recent theory for shear-thinning fluids (Picchi et al., Journal of Fluid Mechanics, 2021, 918). Then, we investigate the bubble hydrodynamics far from the validity range of the current models. Finally, we study the scaling of the bubble velocity and lubrication film thickness, extending the current theory to shear-thinning fluids.
\end{abstract}

\section{Introduction}

The study of fluids in a microchannel (i.e., microfluidics) is crucial for the intensification of industrial processes [1] (i.e, decreasing the size of an industrial system retaining the production capacity). Some examples are the biological and chemical microreactors [2, 3], and cooling systems for microelectronic devices [4]. Further applications could include modeling of porous media in oil extraction [5], convective drying [6], and $\mathrm{CO}_{2}$ sequestration [7].

\footnotetext{
*Corresponding author: andrea.aquino@unibs.it
} 
Microchannels present a rectangular or circular cross-section of a sub-millimeter hydraulic diameter [8], and the viscous and surface tension forces dominate over the inertial and buoyancy forces. Since this regime could produce long diffusion times and low mixing performance $[9,10]$; multiphase flows can enhance the heat and mass transfer rates $[11,12$, 13].

Slug flows are frequent in microfluidics [14, 15], and consist of liquid slugs, alternated to bubbles of elongated shape, Taylor bubbles, that occupy almost entirely the cross-section of the channel, and a thin liquid layer, named lubrication film, separates the gas from the tube wall. Since the transport mechanisms change in the different regions of the bubble [16], predicting the bubble shape is crucial to estimate the heat and mass transfer rates.

First studies on elongated bubble hydrodynamics [17, 18] scale the bubble speed with the capillary number $(\mathrm{Ca}=\mu U / \sigma)$ showing that the bubble moves faster than the mean velocity of the flow by the effect of the lubrication film. The theory by Bretherton [19] models the curvature of the bubble front and the lubrication film thickness in relation to $\mathrm{Ca}$. The most recent works model the bubble profile by more sophisticated techniques to extend Bretherton's theory at broader operating conditions as higher $\mathrm{Ca}$ and square-section capillaries [20, 21, 22].

All works mentioned above study the bubble motion in a Newtonian fluid. Shearthinning fluids are non-Newtonian fluids that exhibit a pure viscous behavior [23]; their deformation depends only on the current value of shear stress (the duration of the deformation is irrelevant); the viscosity is maximum at zero shear rate, and it gradually diminishes to the minimum when the shear-rate tends to infinite. These fluids are typical of biological fluids $[24,25]$.

The hydrodynamics of a Taylor bubble in a shear-thinning fluid presents more complexities than in the Newtonian case [26] because the fluid viscosity changes in the bubble surrounding. Although numerous works study the bubble motion in Newtonian fluids, the available literature poorly investigates the relations between the rheological properties and bubble profile, and studies where the continuous phase exhibits a shearthinning behavior are currently missing.

Our numerical analysis aims to fill this gap. We study the motion of a Taylor bubble in a shear-thinning flow at a broad range of operating conditions, including cases with low and high shear rates. To properly model the bubble motion, we need a constitutive model for the nonn-Newtonian fluid. The power-law is the simplest rheological model, and it finds wide application in microfluidics [28, 29, 30, 31]; however, this model presents poor accuracy when applied to a shear-thinning fluid, especially at a low shear rate. Thus, we solve the Carreau constitutive model [32], which includes an additional term for the viscosity at zero shear rate, and, therefore, it overcomes the limitations of the power-law viscosity model [33]. The solution of the Carreau model introduces further novelty in our works because the application of this constitutive equation in microfluidics is scarce: the current studies on the bubble hydrodynamics in Carreau fluids [34, 35, 36, 37, 38, 39] limit their results to a single fluid (carboxymethylcellulose) and are valid below the tested operating conditions.

The case study is a planar microchannel. Starting from simulating the bubble motion in a Newtonian fluid, we check the independence of the solution from the grid and then validate the numerical results against experimental data from the literature. After the validation of the computational domain, we simulate the bubble motion in shear-thinning 
fluids and compare the results with our recent theory [27], valid only for low $\mathrm{Ca}$. Finally, we investigate the bubble hydrodynamics out of the validity range of the theoretical model. Our final goal is to predict the lubrication film thickness and bubble profile in a wide range of $C a$.

\section{Materials and method}

\subsection{Numerical domain}

The numerical domain is a planar microchannel of rectangular cross-section (Fig.1). The length $L$ of the channel is $500 \mu \mathrm{m}$, and the radius $R$ is $50 \mu \mathrm{m}$. As boundary conditions, we impose the symmetry along the horizontal semi-axis of the channel and a velocity profile at the inlet. In the Newtonian cases, the inlet profile is semi-parabolic:

$$
\begin{gathered}
U_{y}=U_{\max }\left[1-\left(\frac{y}{R}\right)^{2}\right] \\
U_{\max }=1.5 U_{\text {mean }}
\end{gathered}
$$

Where Umax and Umean are the center-line and average fluid velocity, respectively. In shearthinning fluids, the profile is obtained from the solution for Carreau fluids presented in [40]. The no-slip condition sets the velocity of the fluid to zero at the downward wall. The outlet presents no constrains.

We solve a structured grid of $n x \times n y$ squared elements. Similar works [41, 42] show that the lubrication film thickness is particularly sensitive to the number of elements along the vertical direction (ny). Thus, we fix $n x=800$ in all simulation cases and vary $n y$ to check the grid independence of the solution. The vertical dimension of each element changes along the radius, increasing the number of mesh in the proximity of the tube wall to optimize the calculation of the lubrication film.

At time zero, the channel contains the liquid phase and a bubble of $200 \mu \mathrm{m}$ length, positioned at $20 \mu \mathrm{m}$ from the inlet. The bubble rear and front are hemispherical with a radius of $40 \mu \mathrm{m}$, and we calculate the initial pressure difference between the bubble and the fluid by the Laplace equation [43].

In the validation cases, the liquid phase is Newtonian $\left(\rho_{l}=1000 \mathrm{~kg} / \mathrm{m} 3, v_{l}=10^{-3} \mathrm{~m}^{2} / \mathrm{s}\right)$, while we model the effective viscosity of shear-thinning fluids using the Carreau model:

$$
\nu_{1}=\nu_{\infty}+\left(\nu_{0}-\nu_{\infty}\right)\left[1+(k \dot{\gamma})^{a}\right]^{\frac{n-1}{a}}
$$

The values $v_{0}, v_{\infty}$ of the zero and infinite shear viscosity, the $\gamma$ shear rate, the $k$ relaxation time, and $a, n$ coefficients change by the operating conditions to investigate (Sect.2.3). In all simulations, the gaseous phase is Newtonian $\left(\rho_{2}=1 \mathrm{~kg} / \mathrm{m}^{3}, v_{2}=1.48 \times 10^{-3} \mathrm{~m}^{2} / \mathrm{s}\right)$ and the surface tension $\sigma=0.07 \mathrm{~N} / \mathrm{m}$ is constant. 

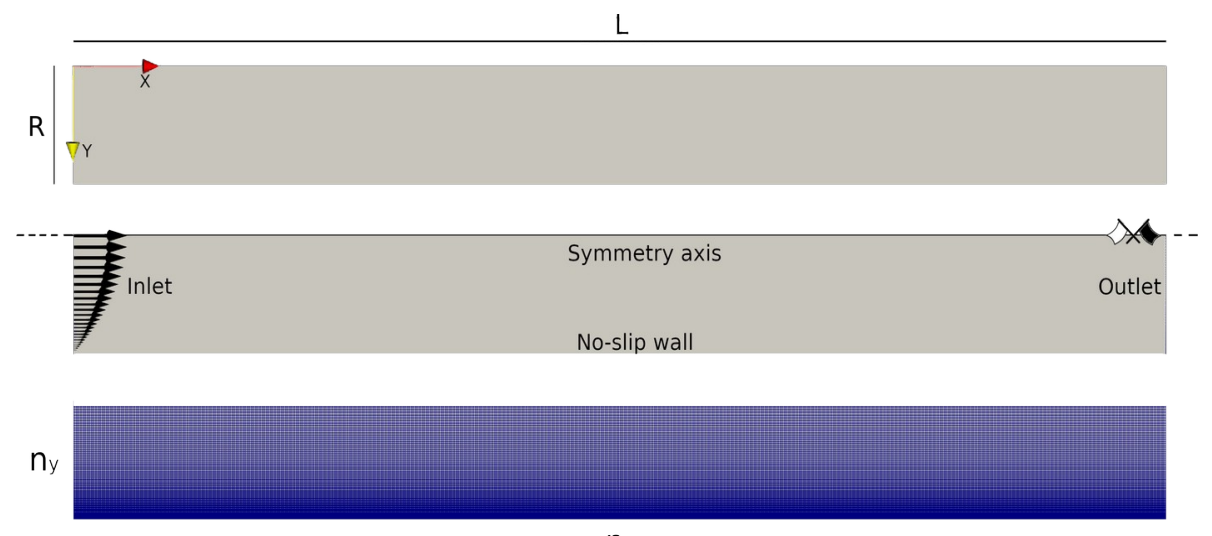

$\mathrm{nx}$

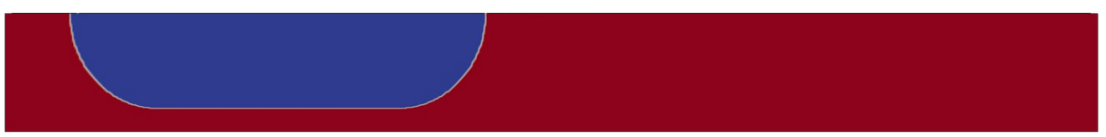

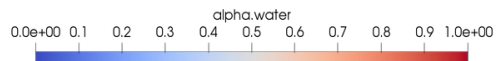

Fig.1 : Different views of the numerical domain; from the top, we can see the microchannel, the boundary conditions, the computational grid, and the volume fraction $\alpha$ of the liquid phase.

\subsection{Governing equations}

We use the interFoam solver of the OpenFOAM-v8. The solved equations represent a system of two immiscible, incompressible, and isothermal fluids, and the interface tracked by the volume of fluid (VOF) technique.

Balance equations of mass and momentum read:

$$
\begin{gathered}
\nabla \cdot U=0 \\
\frac{\partial(\rho U)}{\partial t}+\nabla \cdot(\rho U U)=-\nabla p+\nabla \cdot\left[\mu\left(\nabla U+\nabla U^{T}\right)\right]+\rho g+F_{\sigma}
\end{gathered}
$$

Where $U$ is the fluid velocity, $p$ is the pressure, $g$ is the gravitational acceleration, and $F_{\sigma}$ is the surface tension force. The conservation equations include the contribution of both phases by averaged fluid properties:

$$
\begin{aligned}
& \rho=\rho_{2}+\left(\rho_{1}-\rho_{2}\right) \alpha \\
& \mu=\mu_{2}+\left(\mu_{1}-\mu_{2}\right) \alpha
\end{aligned}
$$

The term $\alpha$ defines the ratio between the volume occupied by the primary phase over the total cell volume, and it varies from 0 and 1 at the interface. In the VOF, the volume fraction $\alpha$ is a passive scalar transported by the flow:

$$
\frac{\partial \alpha}{\partial t}+\nabla \cdot(\alpha U)=0
$$


The discrete solution of this equation needs an appropriate numerical scheme to limit numerical diffusion and ensure the boundedness of the $\alpha$ between 0 and 1. First order schemes preserve the boundedness but generate high diffusion at the interface, the second order schemes are less diffusive but they generate oscillations of the volume fraction out from the definition interval. In our analysis, we solve the transient term by the first order implicit Euler scheme, and the convection term by the Gaussian integration with a linear interpolation (a second order scheme); the VanLeer limiter [44] guarantees the boundedness of $\alpha$. Furthermore, the interFoam solver includes the Multidimensional Universal Limiter with Explicit Solution (MULES) algorithm [45], that balances the effect of numerical diffusion by an artificial compression term in the momentum equation:

$$
\frac{\partial \alpha}{\partial t}+\nabla \cdot(\alpha U)=\nabla\left(\alpha(1-\alpha) U_{r}\right)
$$

The compression term is non-zero only at the interface due to the multiplying factor $\alpha(1-\alpha)$, and the compression velocity $U_{r}$ is:

$$
U_{r}=\min \left[C_{\gamma} \frac{|\phi|}{|S|}, \max \left(\frac{|\phi|}{|S|}\right)\right] \cdot n_{s}
$$

where $S$ is the area of the cell face multiplied by the outward vector ns normal to face, and the term $\varphi=U \cdot S$ is the volume flux accross the cell face. The constant $C_{\gamma}$ is a tuning value which controls the compression; a value of 0 defines no compression, while an higher value ensure a sharp interface. In our simulation, we set $C_{\gamma}=1$ because higher values increase the error of the interface curvature and smearing; this choice is common for simulating surface tension dominated flows (see [46,47]).

\subsection{Operating conditions and dimensionless groups}

As first step to investigate the bubble motion in the Carreau fluid, we compare our numerical results with the recent lubrication model for shear-thinning fluids in [27]. In this theory, the liquid phase follows the Ellis constitutive equation [33], and we study the bubble motion at different degrees of shear-thinning, measured by the Ellis number $E l$. As defined in [27], $E l$ is the ratio between the characteristic shear-rate of the fluid and the representative shear-rate in the lubricating film. When $E l$ tends to infinite, the viscosity model reduces to Newtonian; at small $\mathrm{El}$, the shear-thinning effects dominate.

The above theory is valid as $C a$ tends to zero. Thus, we use it to validate our results at low capillary numbers, and then we investigate broader operating conditions by pure numerical solutions of the bubble motion. We simulate the Taylor bubble in a shearthinning fluid at three orders of magnitude of $\mathrm{Ca}$. At each capillary number, we investigate four rheologies (i.e., four combinations of the Carreau's input parameters), each one corresponding to a specific $E l$ value, so to space from Newtonian to pure shear-thinning behavior. See the simulated rheology curves in Fig.2 and the input parameters of the Carreau model in Table1. 


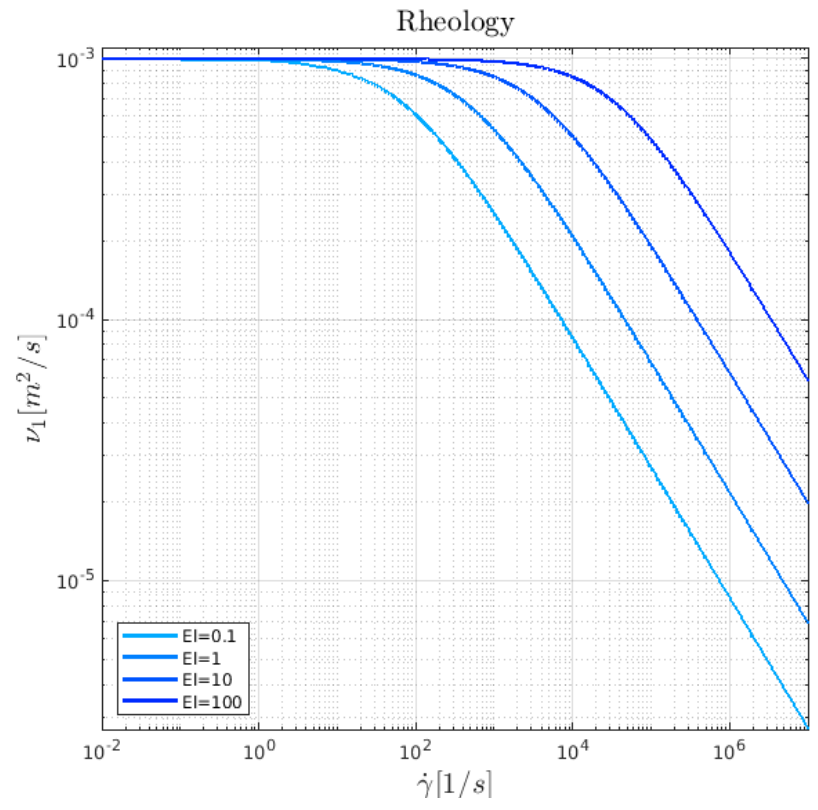

Fig.2 : The investigated rheological curves at $\mathrm{Ca}=10^{-3}-10^{-2}-10^{-1}$.

Table. 1. The input parameters of the Carreau model adn the corresponding Ellis number.

\begin{tabular}{|c|c|c|c|c|c|c|}
\hline \multirow[t]{2}{*}{$\mathbf{C a}$} & \multirow[t]{2}{*}{ El } & \multicolumn{5}{|c|}{ Carreau parameters } \\
\hline & & $\mathbf{n}$ & $\mathbf{a}$ & $v_{0}$ & $\boldsymbol{v}_{\infty}$ & $\mathbf{k}$ \\
\hline \multirow[t]{4}{*}{$10^{-1}$} & 100 & \multirow{12}{*}{0.5} & 0.841 & \multirow{12}{*}{0.001} & \multirow{12}{*}{0} & 0.0138 \\
\hline & 10 & & 0.843 & & & 0.0021 \\
\hline & 1 & & 0.855 & & & 0.0002 \\
\hline & 0.1 & & 0.900 & & & 0.00003 \\
\hline \multirow[t]{4}{*}{$10^{-2}$} & 100 & & 0.841 & & & 0.0297 \\
\hline & 10 & & 0.842 & & & 0.0046 \\
\hline & 1 & & 0.849 & & & 0.0005 \\
\hline & 0.1 & & 0.880 & & & 0.00006 \\
\hline \multirow[t]{4}{*}{$10^{-3}$} & 100 & & 0.840 & & & 0.0559 \\
\hline & 10 & & 0.841 & & & 0.0087 \\
\hline & 1 & & 0.840 & & & 0.0010 \\
\hline & 0.1 & & 0.841 & & & 0.0001 \\
\hline
\end{tabular}




\section{Results and discussion}

\subsection{Grid check and validation of the numerical domain}

This section presents the grid independence check and validation of our numerical solutions against current theories for Newtonian fluids.

We check the grid independence by the film thickness calculated at $C a=10^{-3}$ by grids with different $n_{y}$ values. Simulations at such $C a$ demand the highest level of accuracy (and computational efforts) because the lubrication film typically is two orders of magnitude thinner than the tube radius when $\mathrm{Ca}$ tends to zero [19, 41]. Results (Fig.3) show the progressive stabilization of $\mathrm{H}_{\infty}$ in finest grids $\left(n_{y}=225-250\right)$, where it converges to the theoretical value from Bretherton [19], with a percentage error within 6-8\%. The accuracy augments by the number of mesh; however, too high $n_{y}$ values lead to unfeasible computational costs. Therefore, we locally refine the grid near the tube wall by different expansion rates, namely, the ratio of the coarsest over the thinnest mesh length along the radial direction $\left(\delta_{1} / \delta_{n y}\right.$ in Fig.4). Results show the most severe expansion rates $\left(\delta_{1} / \delta_{n y} \geq 105\right)$ resulting in the lowest percentage error $(5.8 \%)$.

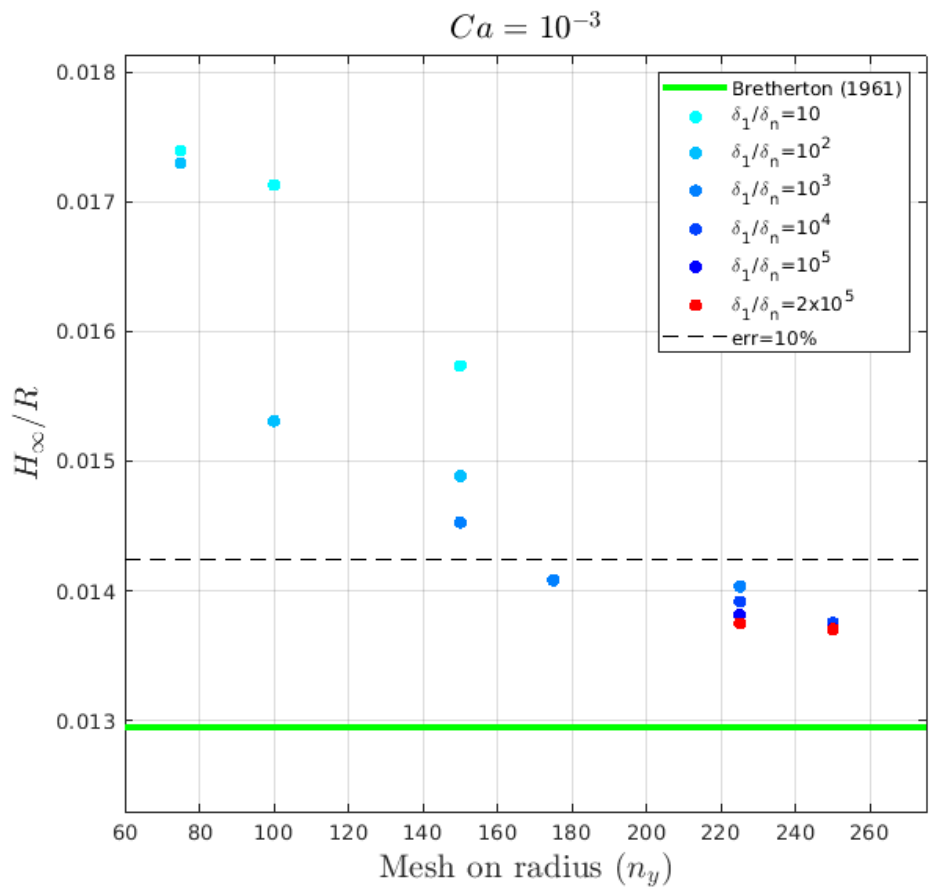

Fig.3: We check the independence of our solution from the grid by calculating the lubrication film thickness with different numbers of elements along the radial direction $\left(n_{y}\right)$ and mesh expansion rate. 
$\delta_{1}$

$\delta n_{y}$

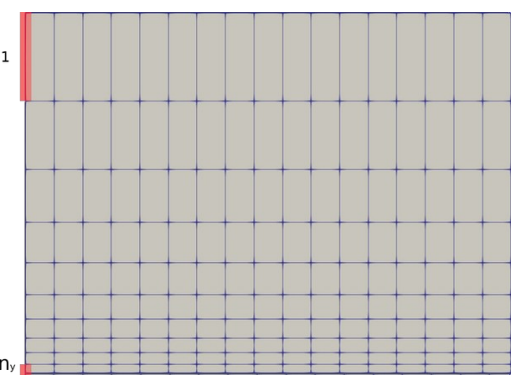

Fig.4: The mesh expansion ratio $\delta_{1} / \delta_{n y}$ is the ratio between the length of the largest $\delta_{1}$ and thinnest $\delta_{n}$ mesh in the radial direction (red highlighting).

The validation compares the dimensionless film thickness obtained from simulations against the theory by Bretherton and results show an excellent agreement at low capillary numbers (Fig.5), with a maximum difference of 8.7\%. Our model diverges from theory when increasing the $\mathrm{Ca}$, and it presents a maximum difference of $20 \%$ at $\mathrm{Ca}=2.4 \times 10^{-2}$. This trend is reasonable because Bretherton's theory, valid when $\mathrm{Ca}$ tends to zero, loses accuracy when the capillary number increases. At high $\mathrm{Ca}$, we compare our results against the correlations proposed by Balestra et al. [48]. The results confirm the quality of our predictions: at $C a \leq 3 \times 10^{-3}$, the percentage error compare to theory is round 5\%, while at higher $\mathrm{Ca}$ is less than $1 \%$.

Finally, we calculate the bubble speed in Newtonian fluid (Fig.6). The bubble moves faster than the liquid flow $\left(U_{b} / U_{f}>1\right)$, and its relative velocity tends to increase with the lubricating film thickness. This trend is following the theory (Eq.12), from which our solutions diverge by less than $1 \%$.

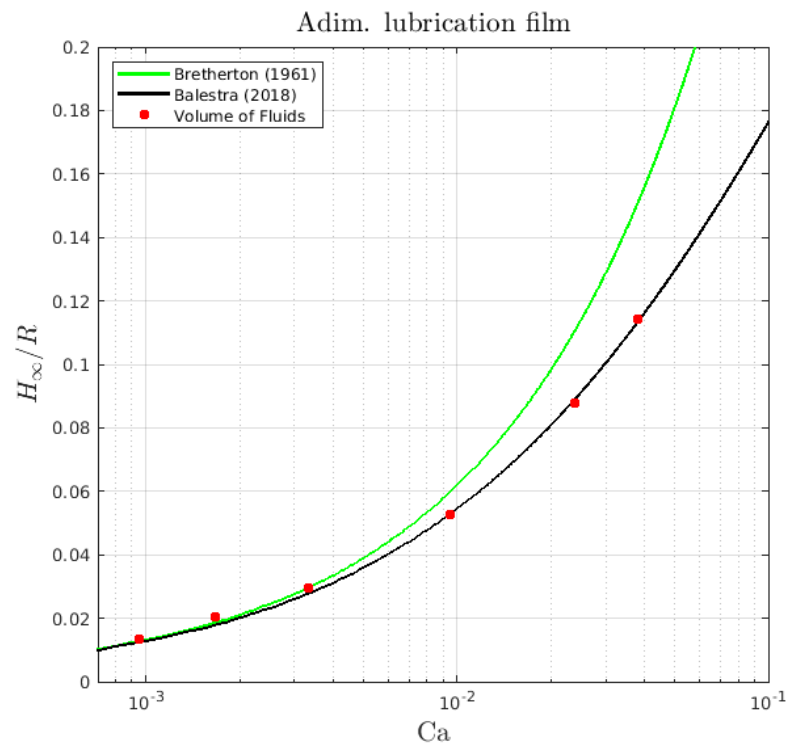

Fig.5:

Comparison between numerical results (red dots) against the current theories for bubble motion in Newtonian fluids : Bretherton's theory (green line) and Balestra et al. [48] (black line). 


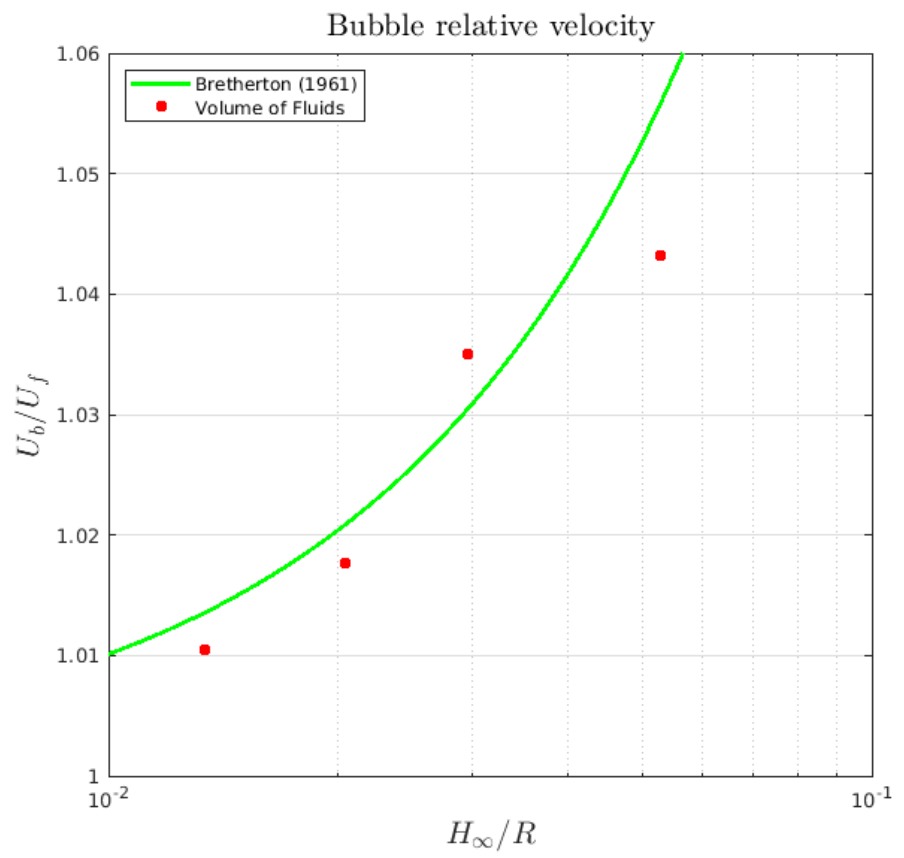

Fig. 6 :

The simulated bubble speed in relation with the lubricating film thickness (red dots) compared to the theoretical trend of [19].

\subsection{Taylor bubble in a shear-thinning fluid}

Fig.7 resumes the profiles of the Taylor bubble simulated in Newtonian and Carreau fluid. In non-Newtonian cases, we change the input parameters of the Carreau model so to investigate the rheological curves shown in Fig.2. Each curve corresponds to a specific Ellis number defined in [23]; in particular, we investigate $E l=0.1-1-10-100$.

Increasing $\mathrm{Ca}$, the bubble change shape assuming from elliptical to elongated shape (see the well-known from Taylor's experiments [18]); the air tends to expands preferably along the center-axis with a consequent thickening of the lubricating film. When $E l$ reduces at fixed $\mathrm{Ca}$, the bubble seems to maintain the equivalent Newtonian shape. However, the lubricating film (Figs.8-9) becomes thinner when the fluid accentuates its shear-thinning behavior. More in general, we observe that the front meniscus assumes a rounded shape accentuating its curvature as $C a$ increases or $E l$ reduces. Such a behavior is coherent to what observed in the simulations with shear-thinning fluids by [26, 35].

The region near the tube wall (i.e., where the fluid is at almost-zero velocity) presents a high-viscosity layer that becomes thinner when $E l$ decreases (i.e., the fluid is losing the Newtonian behavior, reducing the upper plateau in Fig.2). The thickness of this layer is crucial for the final bubble's shape because it directs the expansion of the air towards the the center-axis of the tube.

Finally, we validate the lubricating film thickness at the lowest Capillary numbers against our recent theory [27]. Results show an excellent agreement with theory, presenting at $\mathrm{Ca}=10^{-3}$ and $10^{-2}$ an average error by $11 \%$ and $3 \%$, respectively. 

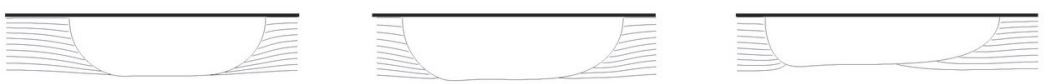

Newt.
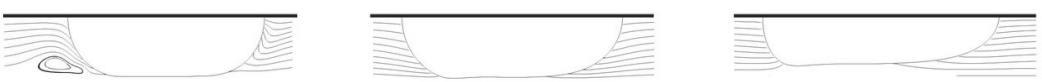

$E \mathrm{I}=100$
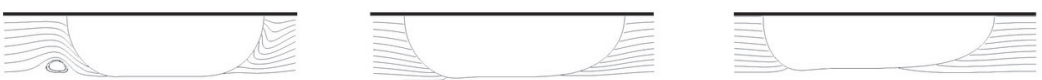

$\mathrm{EI}=10$
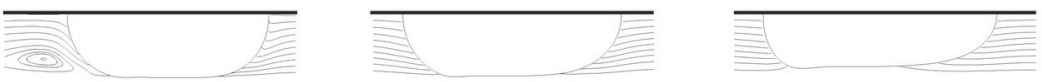

$\mathrm{EI}=1$
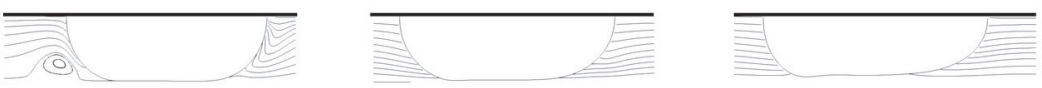

$\mathrm{El}=10^{-1}$

$\mathrm{Ca}=10^{-3}$

$\mathrm{Ca}=10^{-2}$

$\mathrm{Ca}=10^{-1}$

Fig. 7 : The bubble front meniscus in Newtonian and Carreau fluid at $\mathrm{Ca}=10^{-3}$ (right); the numerical solutions vs. theory (left).
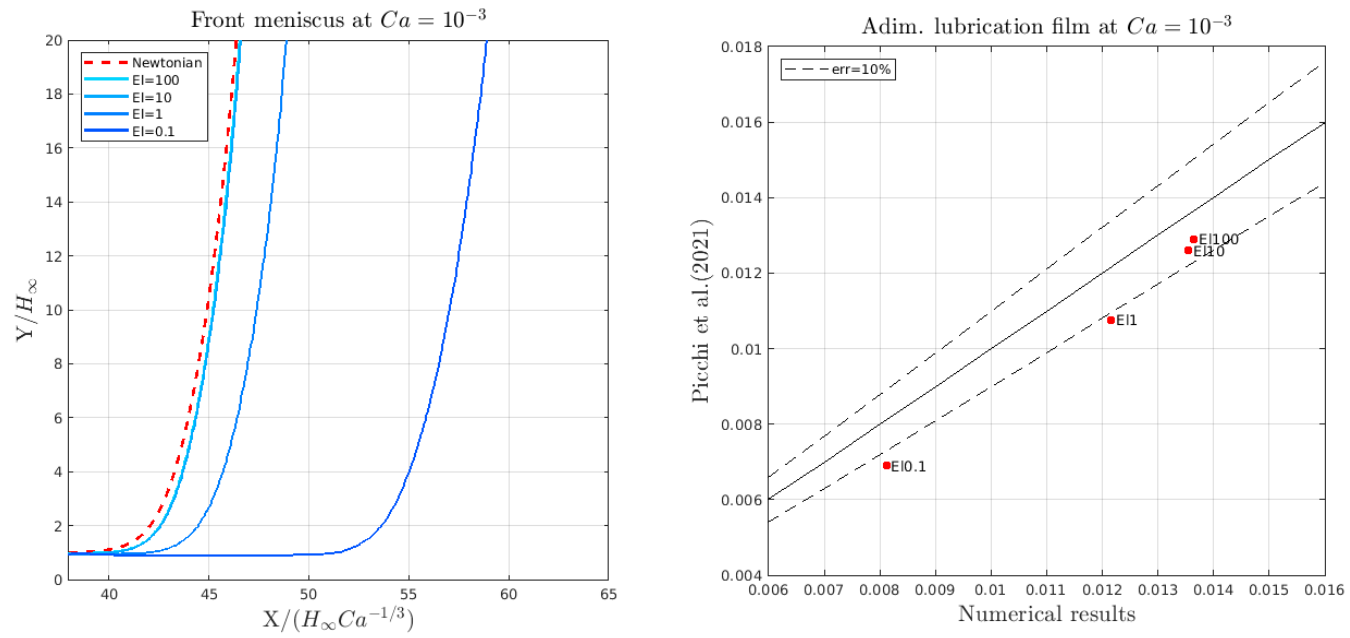

Fig. 8 : The bubble front meniscus in Newtonian and Carreau fluid at $\mathrm{Ca}=10^{-3}$ (right); the numerical solutions vs. theory (left). 

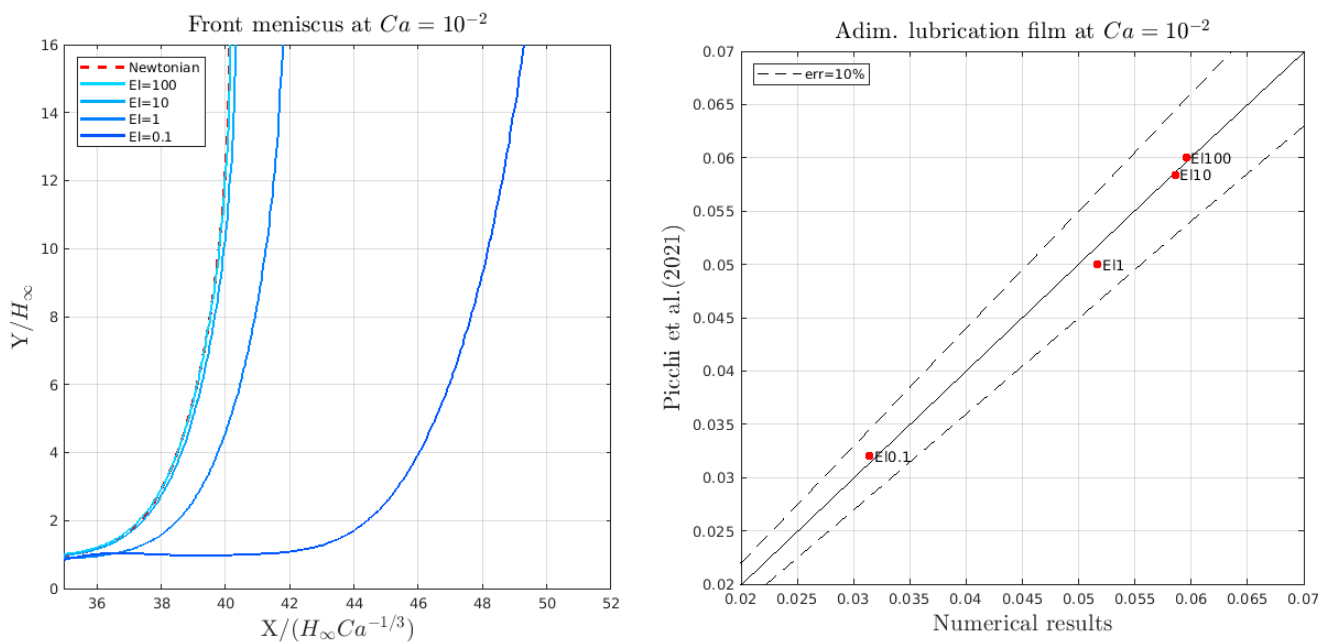

Fig. 9 : The bubble front meniscus in Newtonian and Carreau fluid at $\mathrm{Ca}=10^{-2}$ (right); the numerical solutions vs. theory (left).

\section{Conclusion}

We presented a numerical analysis of a Taylor bubble moving in a shear-thinning fluid. The numerical domain was a planar microchannel with rectangular cross-section. We solved the interFoam equation set, describing the problem as two continuous and incompressible phases, separated by an interface tracked by the volume of fluid technique. First, we checked the independence of the solution from the grid and validated our numerical results against the current theories on the bubble motion in a Newtonian fluid. Then, we described the fluid viscosity by the Carreau model and simulated the bubble motion at different Capillary and Ellis numbers; the latter measures the shear-thinning behavior of the fluid.

Results show the lubricating film thickening and the bubble getting longer with a more accentuated front's curvature as the $C a$ or $E l$ increases. Comparing our results with similar works on shear-thinning fluids, we assumed a high-viscosity layer near the tube wall, where the fluid velocity is almost zero, which becomes thinner when diminishing the $E l$ and $C a$. Such a layer influences the final shape of the bubble, addressing the air expansion toward the central axis of the tube.

Future developments of this work will assess more in detail the formationof such a high-viscosity layer, investigating its dependence on the rheologyand flow conditions and its effects on the lubricating film thickness by theformulation of a scaling law. Further efforts will investigate the formation ofthe bubble profile in all its parts, as the lubricating 
film, the front and rearmeniscus, and their dependence on the bubble velocity and viscosity field,paying particular attention to the phenomena of parasitic currents.

\section{References}

1. A. I. Stankiewicz, J. A. Moulijn, et al., Process intensification: transforming chemical engineering, Chemical engineering progress 96, 22-34 (2000).

2. A. Soulies, J. Pruvost, C. Castelain, T. Burghelea, Microscopic flowsof suspensions of the green non-motile chlorella micro-alga at variousvolume fractions: Applications to intensified photobioreactors, Journalof NonNewtonian Fluid Mechanics 231, 91-101.17 (2016).

3. Y. Ke, W. Zhou, X. Chu, D. Yuan, S. Wan, W. Yu, Y. Liu, Porous copper fiber sintered felts with surface microchannels for methanol steam reforming microreactor for hydrogen production, International Journal of Hydrogen Energy 44, 5755-5765 (2019).

4. T. Bandara, N.-T. Nguyen, G. Rosengarten, Slug flow heat transferwithout phase change in microchannels: A review, Chemical Engineer-ing Science 126, 283-295 (2015).

5. M. Saadat, J. Yang, M. Dudek, G. Øye, P. A. Tsai, Microfluidic investigation of enhanced oil recovery: The effect of aqueous floods and network wettability, Journal of Petroleum Science and Engineering 203, 108647 (2021).

6. A. Aquino, P. Poesio, Off-design exergy analysis of convective dryingusing a two-phase multispecies model, Energies 14, 223 (2021).

7. E. Bonamente, A. Aquino, A. Nicolini, F. Cotana, Experimental analysis and process modeling of carbon dioxide removal using tuff, Sustainability 8, 1258 (2016).

8. S. Kandlikar, S. Garimella, D. Li, S. Colin, M. R. King, Heat transferand fluid flow in minichannels and microchannels, elsevier, 2005.

9. D. J. Beebe, G. A. Mensing, G. M. Walker, Physics and applications of microfluidics in biology, Annual review of biomedical engineering 4, 261-286 (2002).

10. A. D. Stroock, S. K. Dertinger, A. Ajdari, I. Mezi c, H. A. Stone, G. Whitesides, Chaotic mixer for microchannels, Science 295, 647-651 (2002). 
11. S. Wang, Z. Jiao, X. Huang, C. Yang, N. Nguyen, Acoustically inducedbubbles in a microfluidic channel for mixing enhancement, Microfluidicsand nanofluidics 6, 847-852 (2009).

12. A. G unther, M. Jhunjhunwala, M. Thalmann, M. A. Schmidt, K. F.Jensen, Micromixing of miscible liquids in segmented gas-liquid flow, Langmuir 21, 1547-1555 (2005).

13. A. R. Betz, D. Attinger, Can segmented flow enhance heat transfer in microchannel heat sinks?, International Journal of Heat and MassTransfer 53, 3683-3691 (2010).

14. L. Zhao, K. Rezkallah, Gas-liquid flow patterns at microgravity condi-tions, International Journal of Multiphase Flow 19, 751-763 (1993).

15. M. Suo, P. Griffith, Two-phase flow in capillary tubes (1964).

16. M. T. Kreutzer, F. Kapteijn, J. A. Moulijn, J. J. Heiszwolf, Multiphasemonolith reactors: chemical reaction engineering of segmented flow inmicrochannels, Chemical Engineering Science 60, 5895-5916 (2005).

17. F. Fairbrother, A. E. Stubbs, 119. studies in electro-endosmosis. partvi. the "bubble-tube" method of measurement, Journal of the Chemical Society (Resumed) 527-529 (1935).

18. G. Taylor, Deposition of a viscous fluid on the wall of a tube, Journal of fluid mechanics 10, 161-165 (1961).

19. F. P. Bretherton, The motion of long bubbles in tubes, Journal of FluidMechanics 10, 166-188 (1961).

20. J. R. H.-C. Chang, Transport of gas bubbles in capillaries, Phys. FluidsA 1, 1642 (1989).

21. P. Aussillous, D. Qu'er'e, Quick deposition of a fluid on the wall of a tube, Physics of fluids 12, 2367-2371 (2000).

22. M. Grad, C. Tsai, M. Yu, D. Kwong, C. Wong, D. Attinger, Transientsensing of liquid films in microfluidic channels with optofluidic microres-onators, Measurement Science and Technology 21, 075204 (2010).

23. R. Chhabra, Fluid flow, heat, and mass transfer in non-newtonian fluids:multiphase systems, Advances in heat transfer, 23, 187-278 (1993).

24. J. Hron, J. M 'alek, S. Turek, A numerical investigation of flows of shearthinning fluids with applications to blood rheology, International journal for numerical methods in fluids 32, 863-879.19 (2000)

25. M. Guvendiren, H. D. Lu, J. A. Burdick, Shear-thinning hydrogels forbiomedical applications, Soft matter 8, 260-272 (2012). 
26. J. Ara'ujo, J. Miranda, J. Campos, Taylor bubbles rising through flow-ing nonnewtonian inelastic fluids, Journal of Non-Newtonian FluidMechanics 245, 49 66 (2017).

27. D. Picchi, A. Ullmann, N. Brauner, P. Poesio, Motion of a confined bubble in a shear-thinning liquid, Journal of Fluid Mechanics 918, (2021).

28. Q. Zhao, H. Ma, Y. Liu, C. Yao, L. Yang, G. Chen, Hydrodynamics and mass transfer of taylor bubbles flowing in non-newtonian fluids in a microchannel, Chemical Engineering Science 231, 116299 (2021).

29. M. Dziubinski, H. Fidos, M. Sosno, The flow pattern map of a two-phase nonnewtonian liquid-gas flow in the vertical pipe, International journal of multiphase flow 30, 551-563 (2004).

30. M. Laporte, A. Montillet, A. Belkadi, D. Della Valle, C. Loisel, A. Ri-aublanc, J. Hauser, Investigation of gas/shear-thinning liquids flow at high throughput in microchannels with the aim of producing biosourced foam, Chemical Engineering and Processing-Process Intensification 148, 107787 (2020).

31. C.-I. Sun, Y. J. Lin, C.-I. Rau, S.-Y. Chiu, Flow characterization andmixing performance of weakly-shear-thinning fluid flows in a microfluidicoscillator, Journal of Non-Newtonian Fluid Mechanics 239, 1-12 (2017).

32. P. J. Carreau, Rheological equations from molecular network theories, Transactions of the Society of Rheology 16, 99-127 (1972).

33. S. Matsuhisa, R. B. Bird, Analytical and numerical solutions for laminarflow of the non-newtonian ellis fluid, AIChE Journal 11, 588-595 (1965).

34. S. G. Sontti, A. Atta, Cfd study on taylor bubble characteristics in carreauyasuda shear thinning liquids, The Canadian Journal of Chemical Engineering 97, 616-624 (2019).

35. A. I. Moreira, L. A. Rocha, J. Carneiro, J. D. Ara ujo, J. B. Campos, J. M. Miranda, Isolated taylor bubbles in co-current with shear thinnin gcmc solutions in microchannels-a numerical study, Processes 8, 242.20 (2020).

36. W. Y. Fan, S. C. Li, L. X. Li, X. Zhang, M. Q. Du, X. H. Yin, Hydrodynamics of gas/shear-thinning liquid two-phase flow in a co-flow mini-channel: Flow pattern and bubble length, Physics of Fluids 32, 092004 (2020).

37. T. Fu, L. Wei, C. Zhu, Y. Ma, Flow patterns of liquid-liquid two-phase flow in non-newtonian fluids in rectangular microchannels, Chemical Engineering and Processing: Process Intensification 91, 114-120 (2015).

38. V.-L. Wong, K. Loizou, P.-L. Lau, R. S. Graham, B. N. Hewakandamby, Characterizing droplet breakup rates of shear-thinning dispersed phase in microreactors, Chemical Engineering Research and Design 144, 370-385 (2019). 
39. B. Rostami, G. L. Morini, Generation of newtonian and non-newtonian droplets in silicone oil flow by means of a micro cross-junction, International Journal of Multiphase Flow 105, 202-216 (2018).

40. D. Picchi, P. Poesio, A. Ullmann, N. Brauner, Characteristics of stratified flows of newtonian/non-newtonian shear-thinning fluids, International Journal of Multiphase Flow 97, 109-133 (2017).

41. A. Hoang, Breakup of confined droplets in microfluidics (2013).

42. D. Hoang, V. Van Steijn, L. Portela, M. Kreutzer, C. Kleijn, Modeling of lowcapillary number segmented flows in microchannels using openfoam,in: AIP Conference Proceedings, American Institute of Physics, 1479, 86-89.

43. C. K. Batchelor, G. Batchelor, An introduction to fluid dynamics, Cambridge university press, 2000.

44. B. Van Leer, Towards the ultimate conservative difference scheme.ii. monotonicity and conservation combined in a second-order scheme, Journal of computational physics 14, 361-370 (1974).

45. H. G. Weller, A new approach to vof-based interface capturing meth-ods for incompressible and compressible flow, OpenCFD Ltd., ReportTR/HGW 4, 35.21 (2008).

46. S. S. Deshpande, L. Anumolu, M. F. Trujillo, Evaluating the perfor-mance of the two-phase flow solver interfoam, Computational science \& discovery $\mathbf{5}$, 014016 (2012).

47. S. Khodaparast, M. Magnini, N. Borhani, J. R. Thome, Dynamics of isolated confined air bubbles in liquid flows through circular microchannels: an experimental and numerical study, Microfluidics and Nanofluidics 19, 209-234 (2015).

48. G. Balestra, L. Zhu, F. Gallaire, Viscous taylor droplets in axisymmetric and planar tubes: from bretherton's theory to empirical models, Microfluidics and Nanofluidics 22, 1-27 (2018). 